\title{
The Klynac: An Integrated Klystron and Linear Accelerator
}

\author{
James M. Potter \\ JP Accelerator Works, Inc., Los Alamos, NM \\ David Schwellenbach, Alfred Meidinger \\ National Security Technologies, LLC, Los Alamos, NM
}

For CAARI

August 5-10, 2012

This work was done by National Security Technologies, LLC, under Contract No. DE-AC52-06NA25946 with the U.S. Department of Energy and supported by the Site-Directed Research and Development Program. 


\section{Klynac Concept}

- The Klynac concept integrates:

- an electron gun,

- a radio frequency (RF) power source,

- a coupled-cavity linear accelerator

- into a single resonant system. 


\section{Klynac Overview}

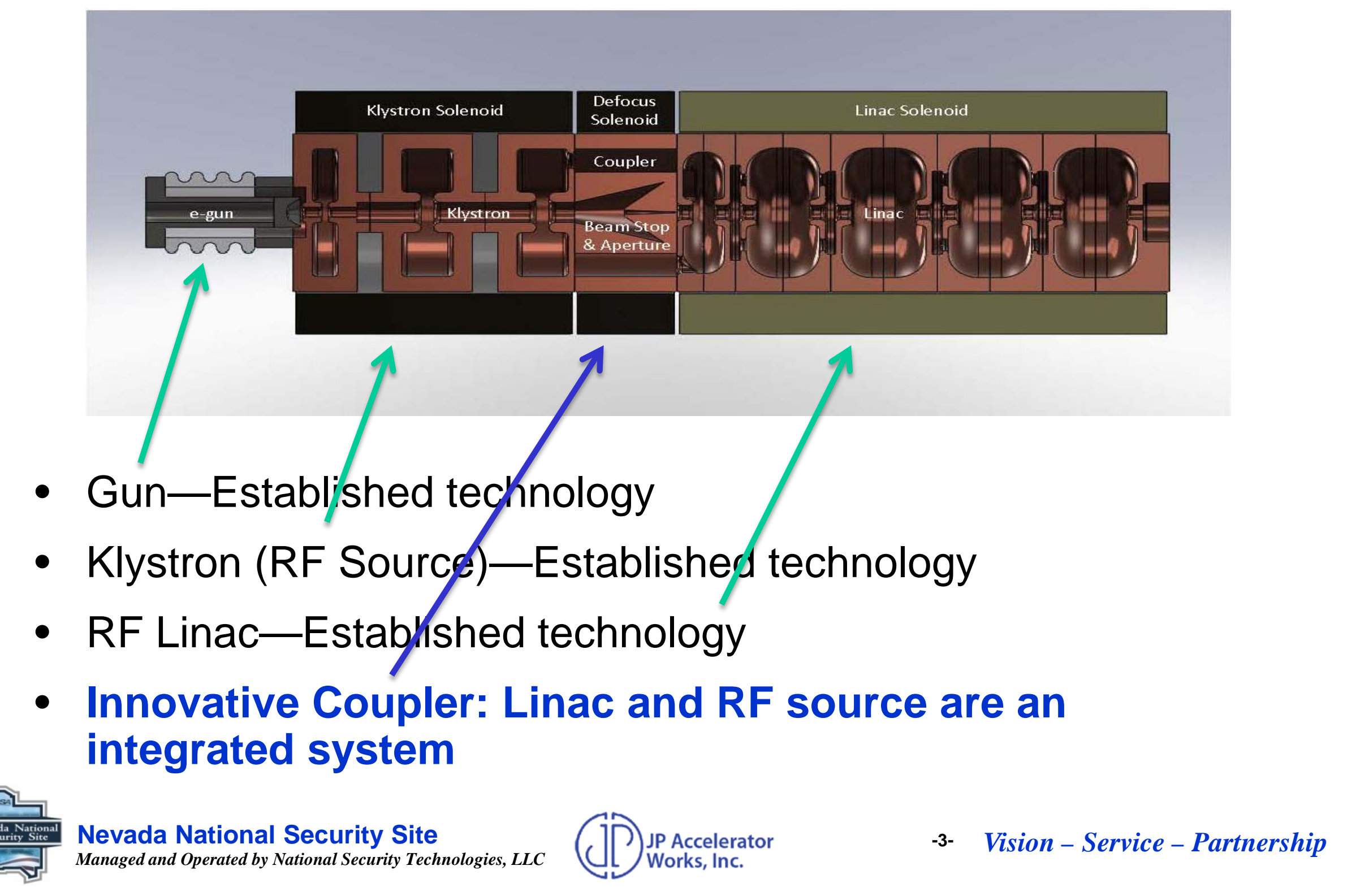




\section{Klynac Coupler}

- The Klynac uses:

- a unique cavity structure to resonantly couple the klystron output cavity to the accelerator cavities,

- locking the two in amplitude and phase,

- and eliminating the normal transmission line between klystron and accelerator. 


\section{Klynac Coupler Concept}

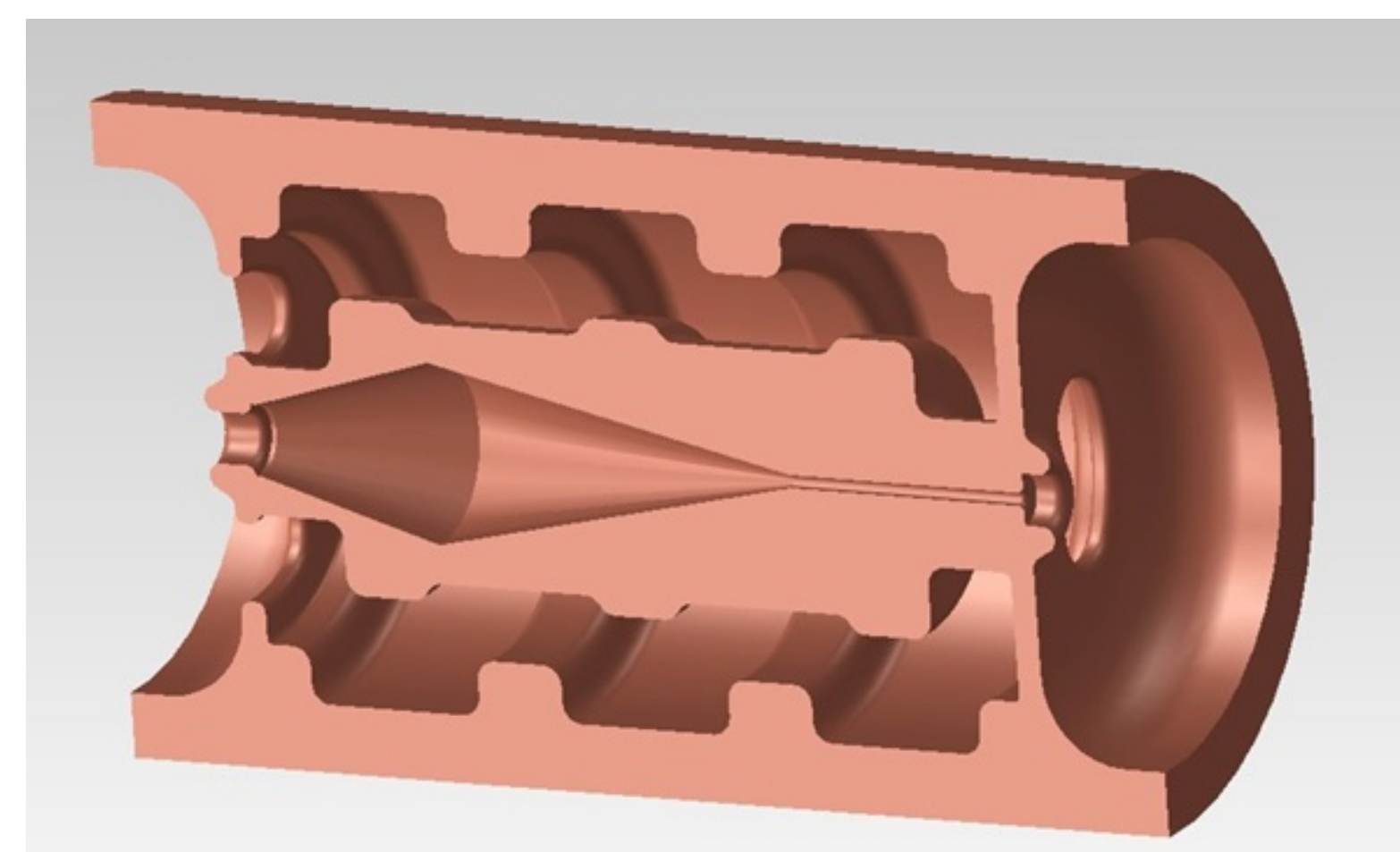

The coupler is a TEM (coaxial) cavity tuned to transmit RF power and resonantly couple the klystron output cavity to the accelerator input cavity 


\section{Resonant Coupling}

- The resonant coupling concept was developed at Los Alamos in the early days of the LANSCE (LAMPF) accelerator design

- The LAMPF design had a coupling resonator that was off axis. It was called a "side-coupled" structure.

- The on-axis coupled accelerator structure was conceived at the same time 


\section{On-Axis Coupled Linac Cell}

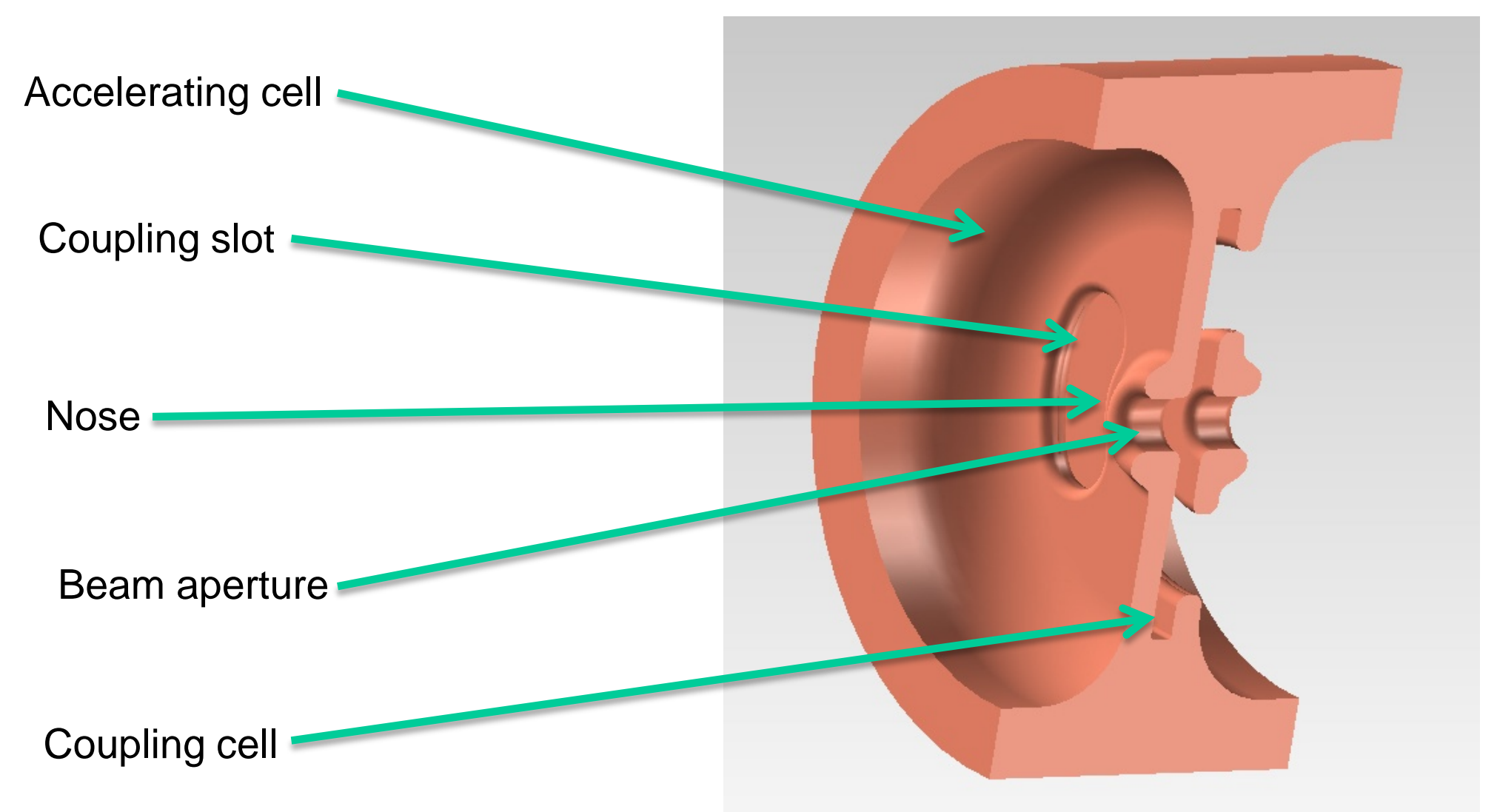

On-axis coupled accelerator structure 


\section{Coupled Circuit Model}

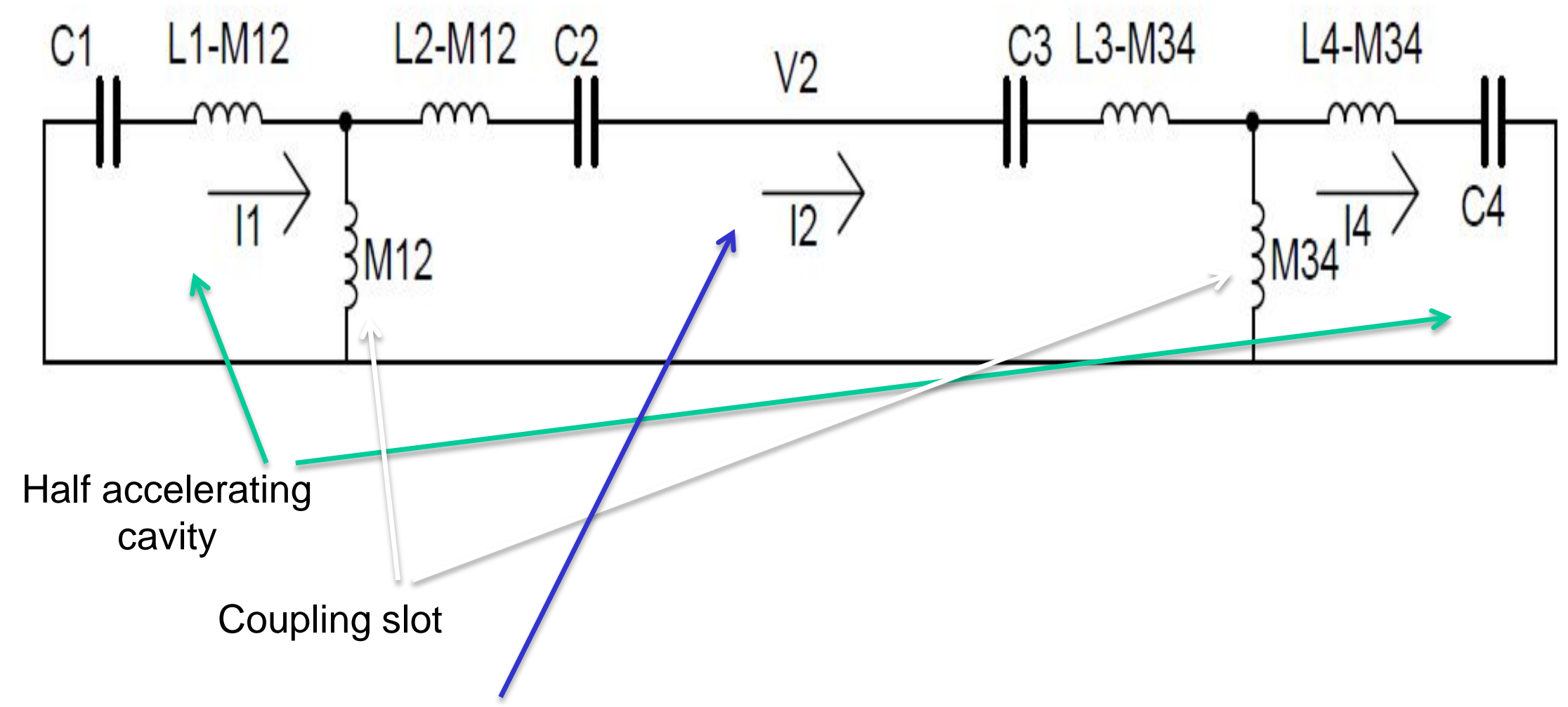

Coupling cavity 


\section{Coupled Circuit Analysis}

- In the perfectly tuned case, the $\pi / 2$ mode frequency is the frequency for which $12=0$.

- This requires that $\omega_{1}=\frac{1}{\sqrt{L_{1} C_{1}}}, \omega_{2}=\frac{1}{\sqrt{L_{2} C_{2}}}$ and $\omega_{1}=\omega_{2}$

- Then the $\pi / 2$ mode frequency is $\omega_{1}$.

- The coupling factors are $k_{12}=\frac{M_{12}}{\sqrt{L_{1} L_{2}}}$ and $k_{34}=\frac{M_{34}}{\sqrt{L_{3} L_{4}}}$.

- The currents I1 and 14 have the opposite sense and $k_{34} I_{4}=-k_{12} I_{1}$. 


\section{Transmission Line Added}

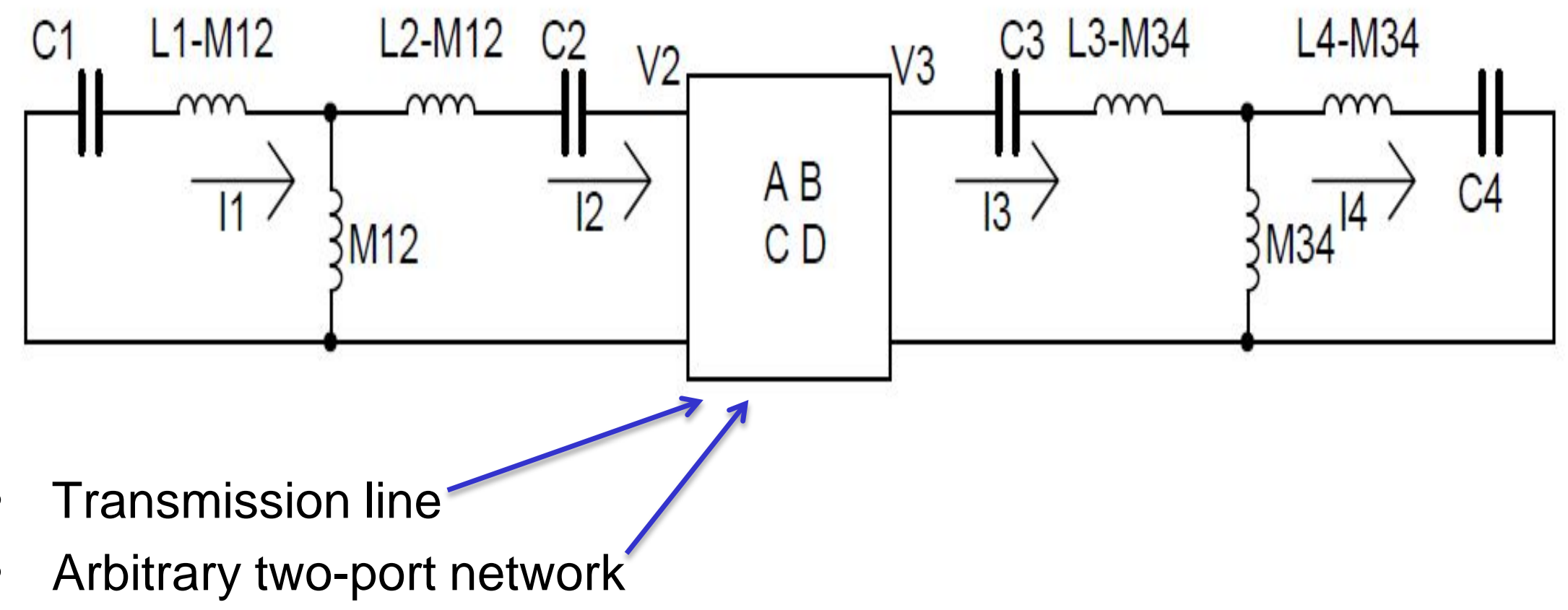




\section{Chain Matrix Representation}

$$
M=\left|\begin{array}{ll}
A & B \\
C & D
\end{array}\right|=\left|\begin{array}{cc}
\cos (\vartheta) & j Z_{0} \sin (\vartheta) \\
\frac{j}{Z_{0}} \sin (\vartheta) & \cos (\vartheta)
\end{array}\right|
$$

- Chain matrix representation of coaxial transmission line

- $\vartheta$ is the electrical length of the line

- $\ell$ is the physical length of the line

- $Z_{0}$ is the characteristic impedance of the line $\vartheta=\frac{\omega \ell}{c}$ 


\section{If Line Electrical Length is a Multiple of $\pi$}

$$
M=\left|\begin{array}{cc}
(-1)^{N} & 0 \\
0 & (-1)^{N}
\end{array}\right|
$$

- $\vartheta=N \pi$

- The amplitude relation is now $k_{34} I_{4}=-(-1)^{N} k_{12} I_{1}$ 


\section{More General Case}

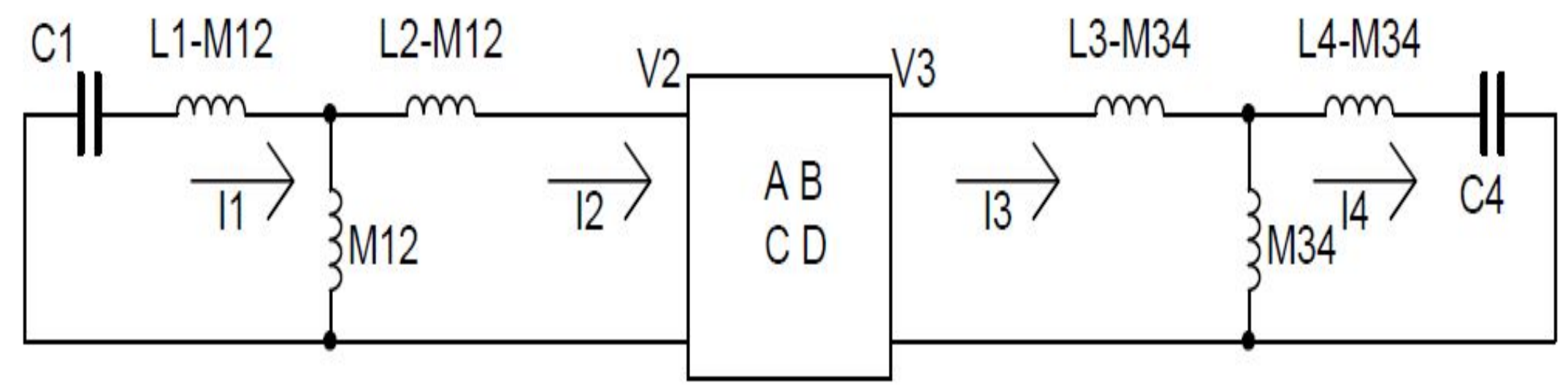

- C2 and C3 have been removed

- For I2 = I3 = 0 we get $M=\left|\begin{array}{cc}1 & -j \omega_{1}\left(L_{2}+L_{3}\right) \\ 0 & 0\end{array}\right|$ 


\section{Form of the Matrix M}

- The matrix M must contain a series impedance that resonates with the coupling slot self inductances at frequency $\omega_{1}$.

- This could be the equivalent of the circuit of Slide 10, with series capacitances and a transmission line of electrical length $\vartheta=N \pi$.

- Or, it could be a more complex circuit. 


\section{Coupler Network Properties}

- The network has to have the property that the currents $12=13=0$ in the accelerating $\pi / 2$ mode, $\omega_{\pi / 2}=\omega_{1}$ and the currents $I 1=14=0$ in the coupling $\pi / 2$ mode, $\omega_{\pi / 2}^{\prime}=\omega_{1}$.

- The accelerating mode is calculated with short circuit boundaries as illustrated in Slide 13.

- The coupling mode is calculated with open circuit boundaries. 


\section{Circuit Model with Open Circuit Boundaries}

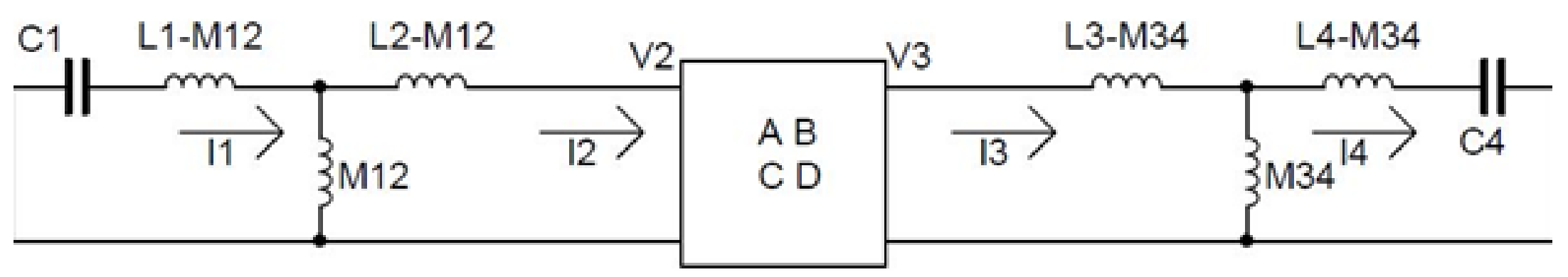

For a periodic structure the resonant frequency is the mode with stored energy in the coupling mode and no stored energy in the accelerating mode 


\section{HFSS Model of a Coupler}

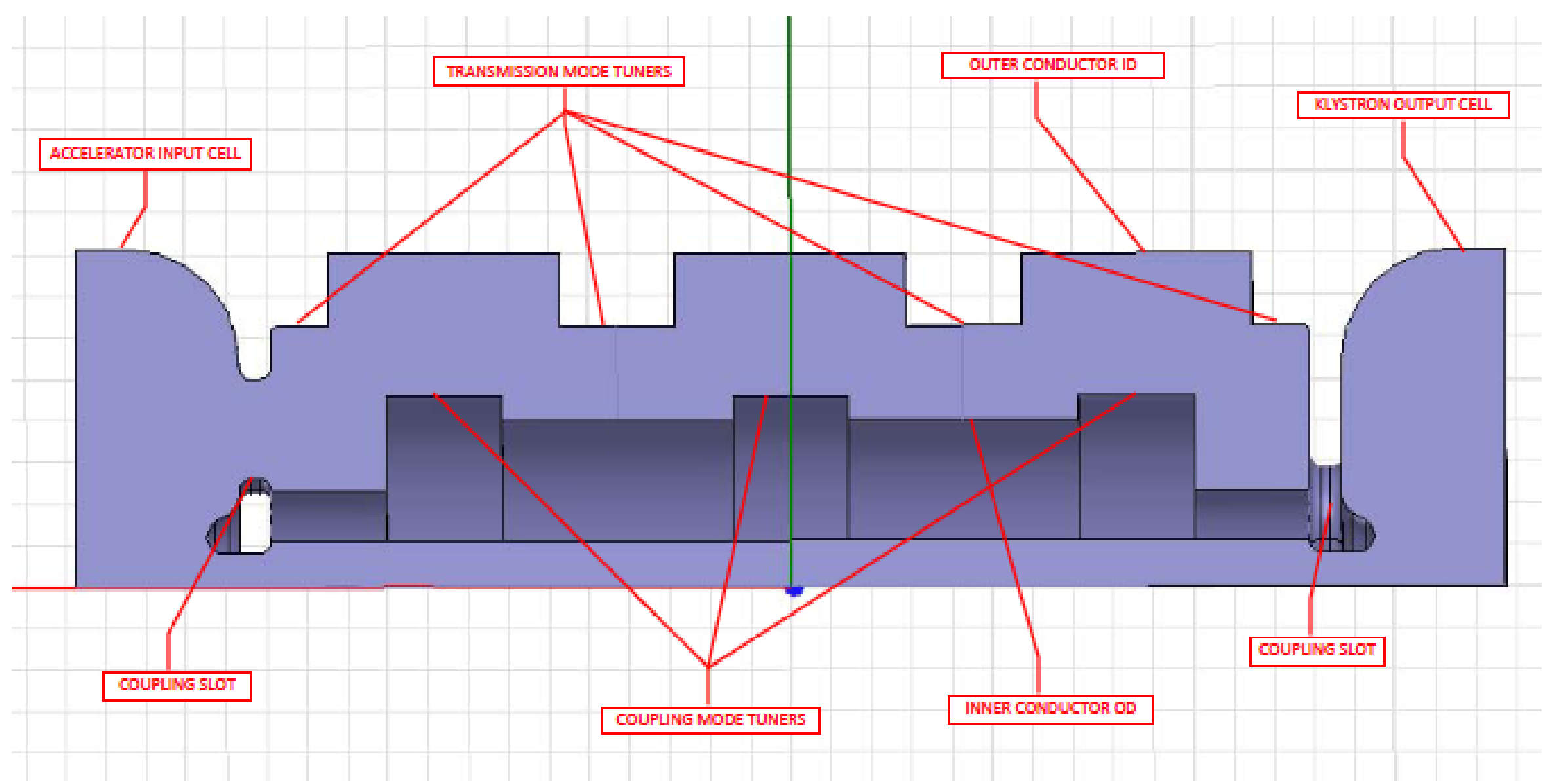

Nevada National Security Site

Managed and Operated by National Security Technologies, LLC

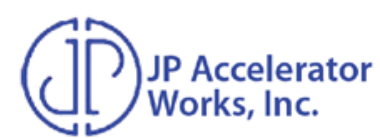

-17- Vision - Service - Partnership 
- The rings on the outer conductor labeled transmission mode tuners capacitively load the coaxial line so that the short circuit mode resonates at the operating frequency

- The rings on the inner conductor labeled coupling mode tuners capacitively load the coaxial line so that the open circuit mode resonates at the operating frequency 


\section{Tilt Sensitivity Method 1}

- The tuning of the accelerating mode can be done by direct measurement

- The coupling mode is tuned by an indirect measurement

- The relative amplitudes of the resonators on each side of the coupler is measured with the resonators detuned 


\section{Tilt Sensitivity Measurement 2}

- One measurement is made with one end tuned high and the other end tuned low keeping the frequency constant

- Then the tuning errors are reversed

- The change in the amplitude ratio is plotted against the frequency of the coupling mode

- When reversing the error produces no change in the field ratio the coupler is tuned 


\section{Coupling Mode Frequency}

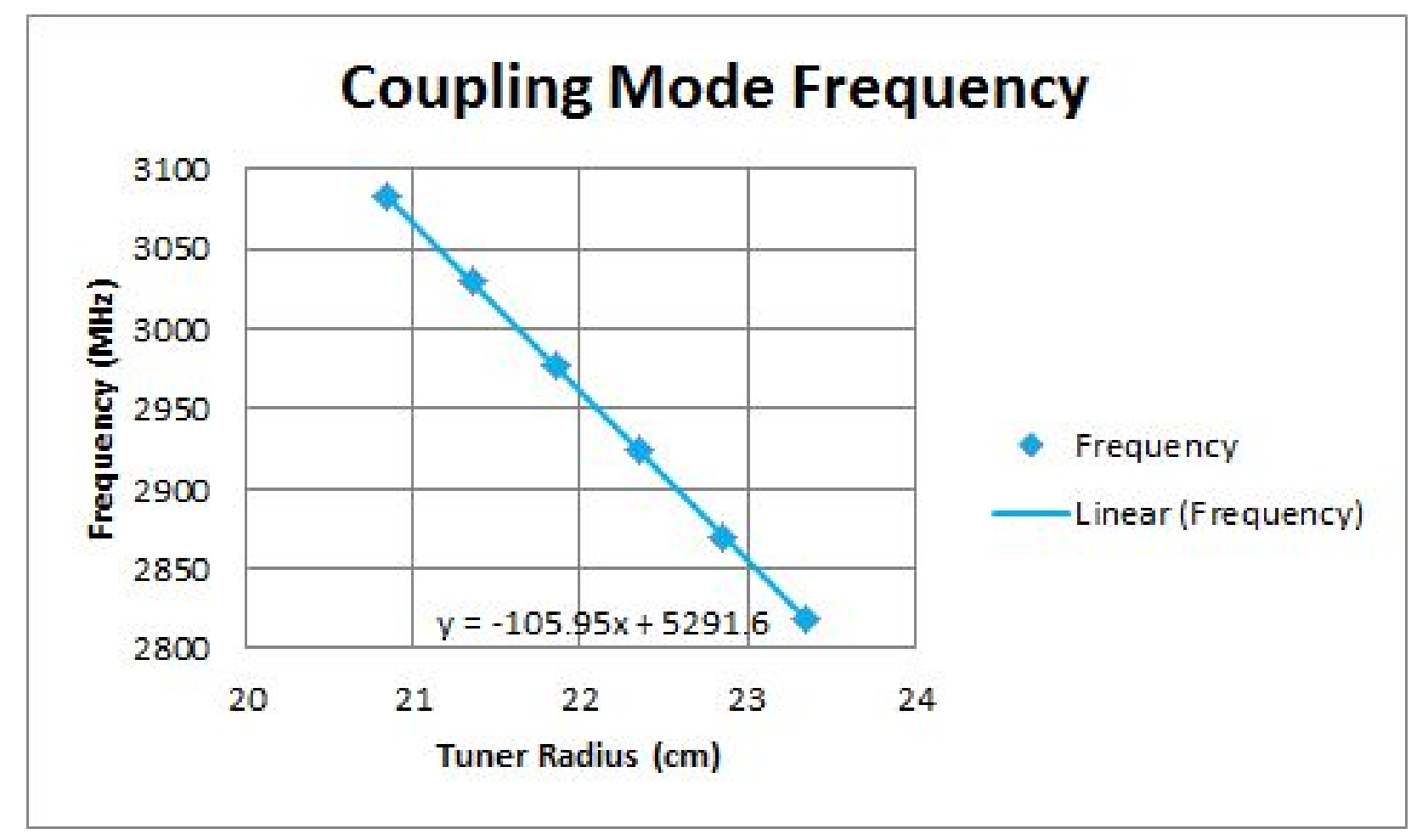

The coupling mode frequency can be calculated with HFSS as a function of tuner radius. 


\section{Tilt Sensitivity versus Coupler Frequency}

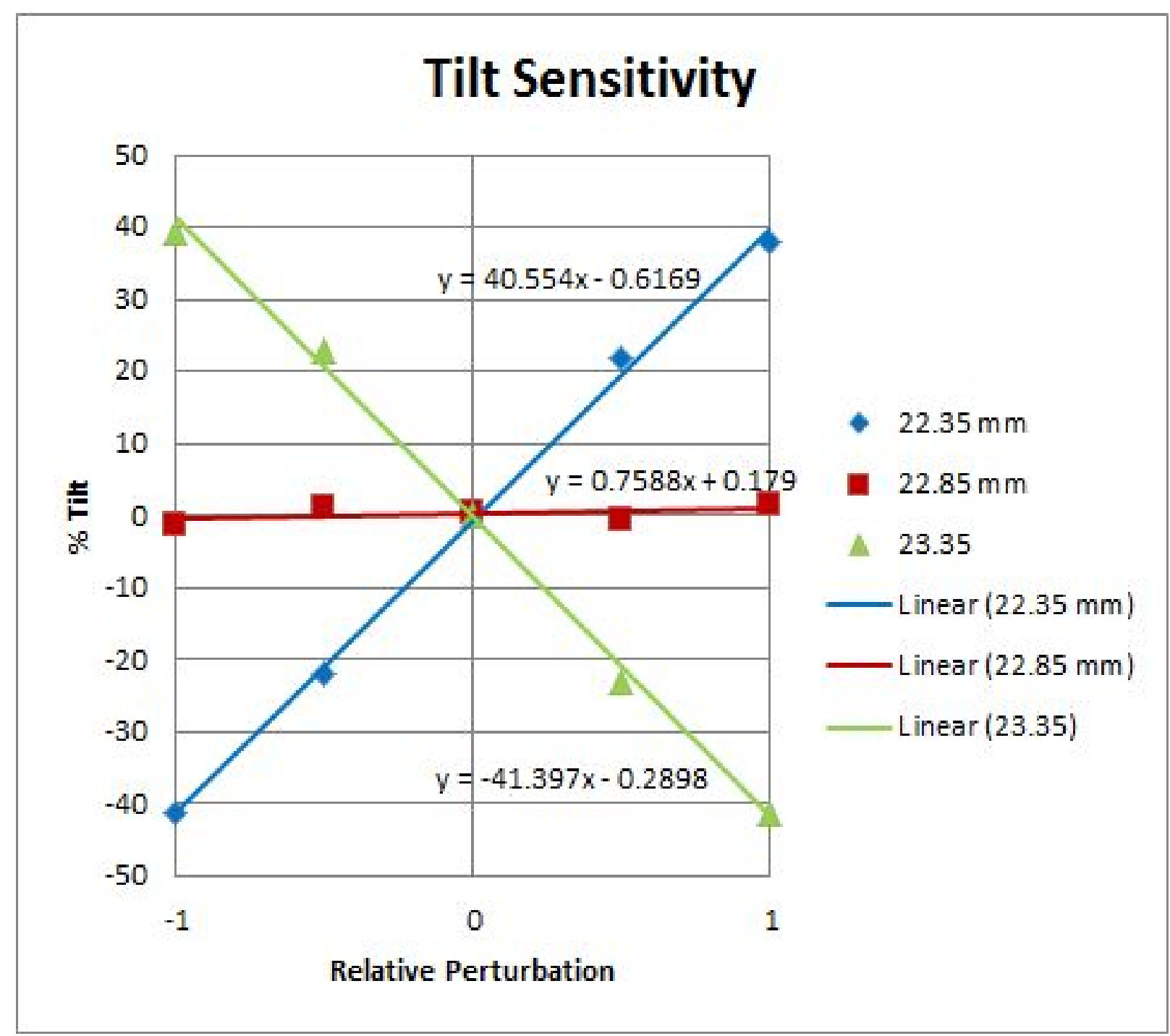




\section{Tilt Sensitivity Technique}

- For several values of the coupler frequency the tilt sensitivity is measured for several tuning errors

- For moderately small tuning errors the tilt sensitivity is linear in the tuning error

- It is also linear in the error in the coupler frequency

- The tilt sensitivity vanishes when the coupler is tuned 


\section{E-Fields in Operating Mode}

- Logarithmic field plot

- Quadrant with symmetry planes

- Half cells at ends

- Short circuit end boundaries

- Equal amplitudes in end cells

- Voltage across slots equal at each end

- Open circuit transmission line mode

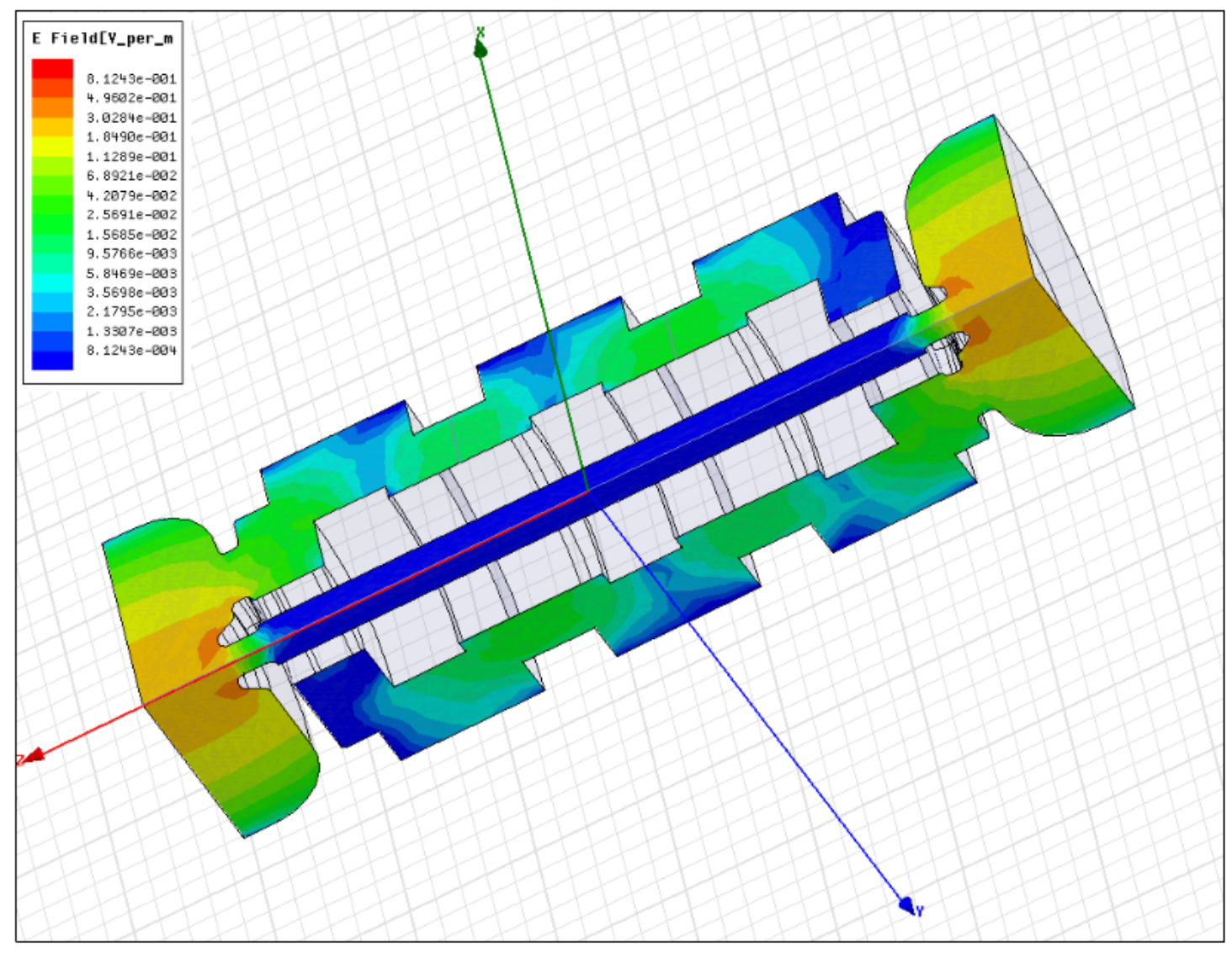




\section{E-Fields in Coupling Mode}

- Logarithmic field plot

- Open circuit boundaries

- Transmission line terminated by slot self inductance

- Mode must be at same frequency as main mode for resonant coupling

- Resonant coupling

- Reduces sensitivity of field distribution to tuning errors

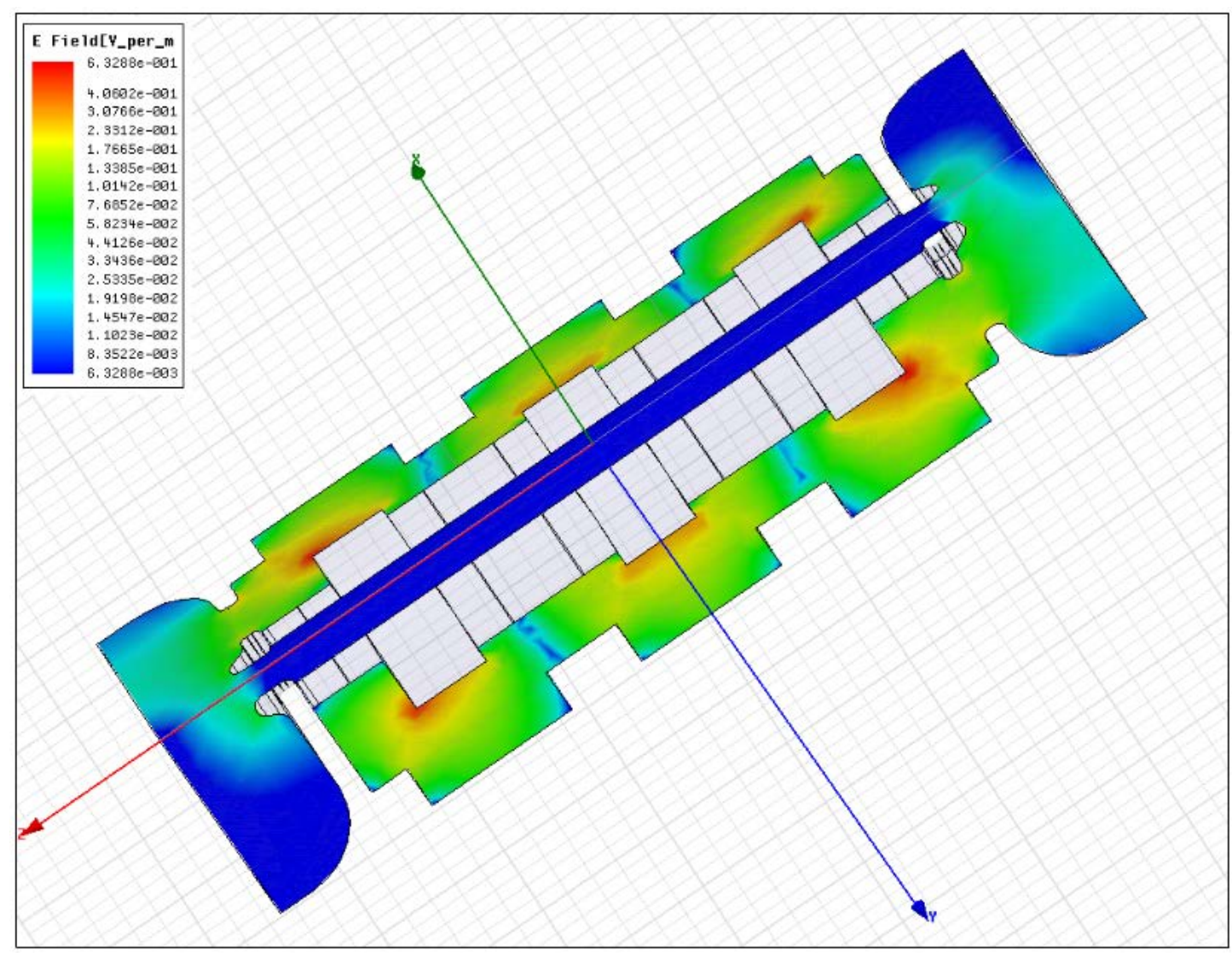




\section{What Next?}

- We have demonstrated that we can make a suitable resonant power coupler of whatever length is required

- We need to do beam dynamics calculations to show that we can get some fraction of the klystron beam to the accelerator at the right phase

- Then we build a cold model to perfect tuning techniques

- Next we would build a full prototype to demonstrate performance 